\title{
Nomenclature for Traumatic Palpebro-Corneo-Conjunctival Adhesions
}

\section{Bhartendu Shukla*}

Ratan Jyoti Netralaya Ophthalmic Institute, Gwalior, Madhya Pradesh, India

*Corresponding author: Bhartendu Shukla, Director of Research and Training, Ratan Jyoti Netralaya Ophthalmic Institute, Gwalior, Madhya Pradesh, India, Tel: +0751-2423350/1; E-mail: bhartendushukla@yahoo.com

Rec date: July 24, 2017; Acc date: September 08, 2017; Pub date: September 11, 2017

Copyright: $\odot 2017$ Shukla B. This is an open-access article distributed under the terms of the creative commons attribution license, which permits unrestricted use, distribution, and reproduction in any medium, provided the original author and source are credited.

\begin{abstract}
It is human nature not to deviate from accepted norms as we become accustomed to them. Medical science is a fast-growing science which is being practiced at many places and in many different ways. No doubt various societies are formed and many conferences are organized top compare results and to bring a uniformity in coining and defining medical terms.
\end{abstract}

Keywords: Traumatic palpebro-corneo; Conjunctival adhesions; Retinitis pigmentosa; Refractile vitreous; Misnomers; Blepharon

\section{Introduction}

In spite of all these efforts differences in definition and terminology develop. This leads to difficulty in comparing results and understanding each other's view point. There is no centralized organization to enforce use of one term in the same way. This has resulted in misnomers (wrong nomenclatures) which have become common in medical literature. There has been little effort made to rectify them.

To indicate inflammation a suffix 'itis' is added but a degenerative condition is called retinitis pigmentosa [1] and refractile vitreous bodies are termed asteroid hyalitis [2]. Similarly, the words palsy, paresis and paralysis are used without clear cut difference [3]. Human nature is resistant to change. So, we continue to use such terms called 'Misnomers' and even try to justify them. However, in the present paper an attempt has been made to identify and rectify such misnomers used for traumatic palpebro-corneo-conjunctival adhesions (Figure 1).

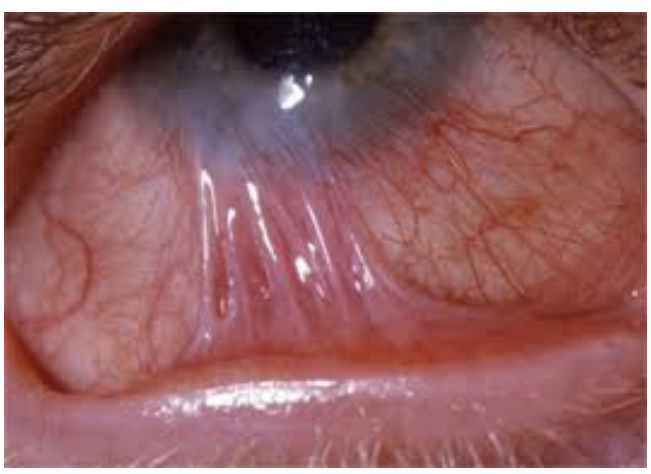

Figure 1: Symconjunctivon (Symblepharon).

\section{Literature Review}

\section{Anatomical considerations}

Conjunctiva is a peculiar structure. Grossly it has palpebral and bulbar parts with superior and inferior fornices. As the palpebral part is firmly attached to the underlying lids and is virtually a part of it. Whereas the fornices and the bulbar conjunctiva are loosely attached to underlying structures and hence they have an independent stature. Thus, adhesion of the palpebral conjunctiva with any structure should be regarded as the adhesion of the lid with that structure. This is not so with bulbar conjunctiva (Figure 2).

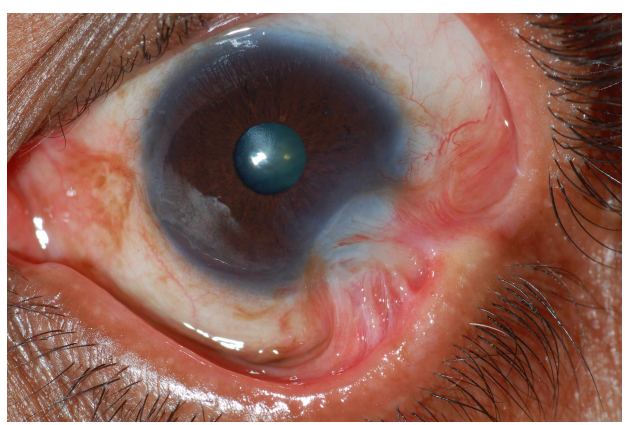

Figure 2: Kerato-conjunctivon (Pseudo-pterygium).

\section{Meaning of some words}

Sym. means together or joined together; ankylos. means stiffening or restriction of movements. Blepharon or palpebrum means lids; keratos means cornea and conjunctiva is the thin layer covering the lids and sclera and making upper and lower fornices. Pseudo means false (Figure 3).

\section{Current terminology}

Symblepharon: Adhesion between bulbar and palpebral conjunctiva; Ankyloblepharon: Adhesion between the lid margins; Pterygium: Adhesion between bulbar conjunctiva and cornea usually on nasal 
side; Pseudo-Pterygium: Adhesion between bulbar conjunctiva and cornea at any meridian.

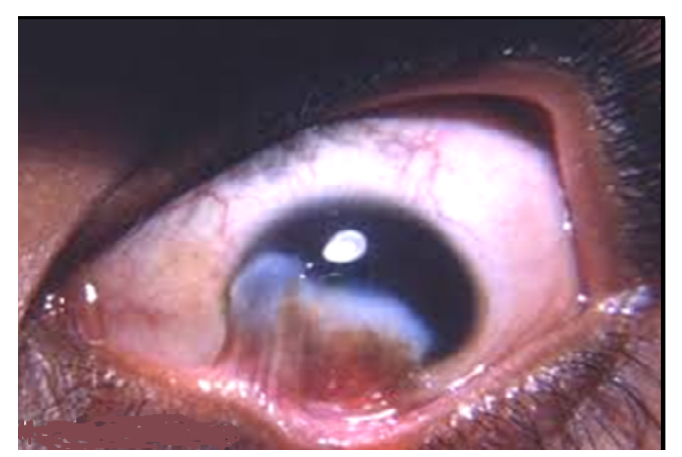

Figure 3: Kerato-blepharon (Adhesion between cornea and lids).

\section{Proposed terminology}

- Symconjunctivon- Adhesion between bulbar and palpebral conjunctiva. It is a union of conjunctivae not of lids.

- Symblepharon- Adhesion between the two lid margins.

- Kerato-conjunctivon- Adhesion between cornea and conjunctiva

- Blepharo-conjunctivon- Adhesion between lids and conjunctiva

- Kerato-blepharon- Adhesion between cornea and lids

- Kerato-blephari-conjunctivon- Adhesion between cornea, lids and conjunctiva

- Symblepharon- filiforme adnate- Adhesion between lid margins by linear adhesions

\section{Discussion and Conclusion}

Old terminology is empirical and the words do not express the underlying pathology hence it causes confusion. The new terminology is scientific, rational and hence should be adopted. It may take some time to get used to the new terminology but truth should not be sacrificed for the sake of convenience. It may be noted that such changes have occurred previously also. The meaning of many words used in ocular trauma have been redefined [4]. A new classification of ocular trauma has recently been published which is very different from the one used since 1996 [5]. Hence any change which is genuine and stands to reason should not be affected by personal likes and dislikes of some individuals. The progress of a subject does not imply only in finding new things but also in rectifying and deleting obsolete things (Figure 4).
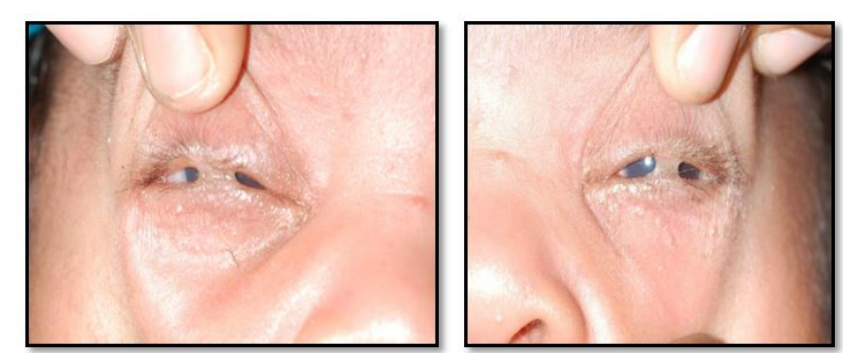

Figure 4: Bilateral Median Symblepharon (Ankyloblepharon).

\section{Acknowledgement}

Some typical photographs are attached for which the author is grateful to Dr. Usha Kim of Aravind Eye Care System, Madurai. I am grateful to Mr. M. Shiva Ram and Mr. C. Majumder for secretarial help. I wish to express my thanks to Dr. Purendra Bhasin and Dr. Priyamvada Bhasin, Directors of Ratan Jyoti Netralaya and Ophthalmic Institutte, Gwalior.

\section{References}

1. Dunbar Roy AB (1895) Retinitis pigmentosa. Ann Ophthalmol 4: 24-28.

2. March W, Shoch D, O'Grady R (1974) Composition of asteroid bodies. Invest Ophthal 13: 701-705.

3. Linder TE, Abdelkafy W, Cavero-vanek S (2010) The management of facial nerve palsy, "paresis" versus "paralysis"and sources of ambiguity in study design. Otol Neurotol 31: 319-327.

4. Kuhn F, Morris R, Witherspoon CD, Heimann K, Jeffers JB, et al. (1996) A standardized classification of ocular trauma. Ophthalmology 103: 240-243.

5. Shukla B, Agrawal R, Shukla D, Seen S (2017) Systemic analysis of ocular trauma by a new proposed ocular trauma classification. Indian J Ophthalmol 65: 718-722. 